\title{
ORIGINAL
}

\section{VALIDACIÓN DE ESCALA PARA EVALUAR LA RELACIÓN FAMILIA-TRABAJO EN MÉDICAS Y MÉDICOS DE FAMILIA (*)}

\author{
Ana Delgado Sánchez (1); Lorena Saletti- Cuesta (1); Silvia Toro- Cárdenas (1); Luis \\ Andrés López- Fernández (1); Juan de Dios Luna del Castillo (2); Inmaculada Mateo- \\ Rodríguez (1)
}

(1) Escuela Andaluza de Salud Pública

(2) Departamento de Bioestadística. Facultad de Medicina. Universidad de Granada

(*)Ayudas concedidas por la Consejería de Salud de la Junta de Andalucía (Expediente: 0020/2006); por el Fondo de Investigación Sanitaria (Expediente PI071176) y los Fondos FEDER de la Unión Europea

\begin{abstract}
RESUMEN
Fundamentos: Estudiar las relaciones familia-trabajo es relevante porque afectan a la vida personal y profesional. El número de mujeres aumenta en medicina sin que se redistribuyan las tareas y responsabilidades domésticas. El objetivo fue elaborar y validar una escala sobre las relaciones familia-trabajo en médicas y médicos de familia (MF) de Andalucía.
\end{abstract}

Métodos: Estudio transversal y multicéntrico. La población de estudio fueron MF de los centros de salud urbanos de Andalucía, muestra $=500 \mathrm{MF}, 50 \%$ por sexo. Estudiamos: sexo, edad, formación postgraduada en medicina de familia, carga asistencial ( 2 variables) y carga familiar ( 7 variables). Diseñamos un cuestionario postal para ser autoadministrado con una escala de 13 preguntas sobre las relaciones familia-trabajo. se realizó análisis bivariante, factorial exploratorio y regresión múltiple para probar la validez convergente.

Resultados: La tasa de respuesta fue del 73,6\%. Se identificaron dos dimensiones: Sobrecarga y Déficit de Apoyo Familiar (SDAF) (5 preguntas) y Conflicto Familia-Trabajo (CFT) (6 preguntas). En ambas las médicas puntúan más alto que sus compañeros. Hay más variables familiares asociadas a ambas dimensiones en las médicas que en los médicos, lo que expresa diferencias en la complejidad de las causas de SDAF y CFT por sexo.

Conclusiones: La escala obtenida es válida, fiable y recoge dos dimensiones empíricas de las relaciones familia-trabajo.

Palabras clave: Médicas de familia. Familia. Trabajo. Escalas.

\section{ABSTRACT}

\section{Validation of Scale to Evaluate the Rela- tionship Between Family- Work in Family Physicians}

Bakcground: Studying the work-family relationships is important because it affects the personal and professional life. Women increases in medicine without redistribute domestic tasks and responsibilities. The purpose of this study was to create and validate a scale of work-family relations in women and men family physician (FP) in Andalusia.

Methods: Cross sectional and multicenter study. The study population were FP of urban primary care centres, sample $=500 \mathrm{FP}(50 \%$ by sex $)$. We studied: sex, age, postgraduate family medicine specialty, care burden ( 2 variables), and family burden ( 7 variables). We design, and included in self-administered questionnaires, a scale of 13 questions on work-family relations. Bivariate analysis, exploratory factor and multiple regressions to test the convergent validity was performed.

Results: The response rate was $73.6 \%$. We identified two dimensions, Overloading and Family Support Deficit (OFSD) (5 questions), and Work-Family Conflict (WFC) (6 questions), in both, female FP score higher than their peers. There are more family variables associated with both dimensions in female FP, it expresses differences in the complexity of the causes of OFSD and WFC by sex.

Conclusion: The scale obtained is valid, reliable and gives two empirical dimensions of family-work relationships.

Key words: Family physician. Family. Work. Scale.

Correspondencia:

Ana Delgado Sánchez

C/Alquería, 66

18010 Granada

España

ana.delgado.easp@juntadeandalucia.es 


\section{INTRODUCCIÓN}

Las desigualdades de género en la profesión médica adquieren progresivamente mayor relevancia debido al incremento de médicas que, en España, pasaron del 1\% en 1955 al $42 \%$ en $2005^{1}$, y en 2009 el $45 \%$ de colegiados en medicina eran mujeres ${ }^{2}$. Pero las médicas no desarrollan su profesión en condiciones de igualdad, tanto fuera como dentro de España encuentran barreras y están infra representadas en comités profesionales y cargos directivos $^{3-6}$, así como en la investigación y en las publicaciones científicas ${ }^{7-9}$.

Las mujeres están accediendo en gran medida a la educación superior y al trabajo remunerado sin que se redistribuyan las tareas y responsabilidades domésticas ${ }^{10}$. Entre médicos y médicas existen desigualdades similares. En Cataluña las médicas dedican más horas al trabajo doméstico ${ }^{5}$. En Andalucía las profesionales sanitarias causan el $73 \%$ de permisos por enfermedad o fallecimiento de familiar ${ }^{11}$.

El estudio de las relaciones familia-trabajo se produce por el aumento de mujeres con trabajo remunerado y por la existencia de interacciones trabajo-familia. Para explicar ambas relaciones se usan teorías centradas en la naturaleza de las mismas ${ }^{12,13}$. En la actualidad se postula un modelo integrador de diversos enfoques teóricos que caracteriza las relaciones familia-trabajo por dos componentes, el conflicto y el apoyo.

El conflicto familia-trabajo se atribuye a la incompatibilidad potencial de roles y demandas en ambas esferas, siendo bidireccional. En esta hipótesis existe una interacción negativa de roles que se interpreta como un conflicto interrol ${ }^{14}$.

Actualmente se plantea que los roles múltiples son beneficiosos o facilitadores ${ }^{15}$, debido en parte al apoyo social que proporcionan y que conlleva un impacto beneficioso en la salud $^{16}$. Una vertiente del apoyo social es el apoyo familiar para el trabajo, que consiste en una serie de conductas de valoración del esfuerzo y en la disposición a prestar ayuda y compartir responsabilidades con la persona que realiza un trabajo remunerado ${ }^{17}$.

Como hemos mencionado, las médicas se responsabilizan de la mayoría del trabajo doméstico, realizando un mayor esfuerzo total de trabajo, esta doble jornada es una doble presencia debido al desempeño sincrónico de roles $^{18}$. La doble presencia puede verse como la inversa del apoyo familiar, al disminuir éste la carga total de trabajo aumenta. Por ello incluimos en el estudio de las relaciones familia-trabajo este componente relacionado con las desigualdades de género.

Estudiar las relaciones familia-trabajo es relevante porque afectan a las decisiones sobre la vida personal y profesional ${ }^{19}$ y se relacionan con resultados como el estrés y otros indicadores de salud ${ }^{20}$. En nuestro medio no disponemos de escalas para medir estas relaciones en profesionales sanitarios. Como parte de una investigación más amplia, nos propusimos construir y validar una escala para medir las relaciones familia-trabajo en médicas y médicos de familia de Andalucía.

\section{SUJETOS Y MÉTODO}

Estudio descriptivo, transversal y multicéntrico.

La población de estudio fueron las médicas y los médicos de familia (MF) que trabajan en los centros de salud (CS) de las capitales andaluzas. Los criterios de inclusión fueron dos, llevar como mínimo un año con el mismo cupo de pacientes y utilizar la historia clínica informatizada de Atención Primaria. Obtuvimos una muestra aleatoria por etapas, primero de $88 \mathrm{CS}$ y segundo de un número de MF acorde con el tamaño del centro: $4 \mathrm{MF}$ en $8 \mathrm{CS}, 5 \mathrm{MF}$ en $12 \mathrm{CS}$, y $6 \mathrm{MF}$ en $68 \mathrm{CS}$, seleccionados/as del listado nominal del centro, resultando una muestra de 500 MF, $50 \%$ por sexo $(\mathrm{alfa}=5 \%$ y potencia del 
$90 \%$ para detectar una diferencia del $15 \%$ entre médicos y médicas). La muestra final fueron 254 médicas y 246 médicos.

Se estudiaron las variables sexo, edad, formación postgraduada en medicina de familia (MIRMF), carga asistencial (mediante número de pacientes en el cupo ajustado por edad y media de pacientes/día en consulta, ambos en noviembre-2007), carga familiar o responsabilidades familiares medida con: situación familiar en dos categorías [vivir solo/a con o sin hijos/as por un lado y resto (vive en pareja con o sin hijos/as; otras situaciones- por otro)], hijos/as en el hogar (dicotómica), horas/día dedicadas a tareas domésticas de lunes a viernes, horas/día dedicadas a tareas domésticas sábados y domingos, quién realiza principalmente las tareas domésticas en tres categorías (yo sola/o, mi pareja, otras situaciones), presencia en el hogar de personas que requieren cuidados especiales (dicotómica: $<15$ años, y/o $>65$ años, y/o personas con discapacidad), y la pareja trabaja fuera del hogar (dicotómica).

Para medir las relaciones trabajo-familia construimos una escala con 13 preguntas referidas a tres dimensiones teóricas: conflicto familia-trabajo: dos preguntas de una escala validada ${ }^{12}$ y cuatro de elaboración propia. Apoyo familiar: dos ítems de una escala validada ${ }^{17}$ y uno de elaboración propia. Y doble presencia: cuatro preguntas del instrumento ISTAS $21^{18}$, adaptadas para su uso en medicina de familia. La escala de respuesta fue tipo Likert con 8 opciones desde "Muy de Acuerdo" a "Muy en Desacuerdo". Las preguntas enunciadas en negativo se recodificaron para el análisis.

Realizamos una prueba piloto con $14 \mathrm{MF}$ para probar la compresión, el orden y las dificultades o resistencias en la respuesta a las preguntas.

Las fuentes de datos fueron a) un cuestionario postal autoadministrado (diciembre-
07 a mayo-08) con todas las variables personales y profesionales, reforzando la respuesta con la recogida en mano. Pedimos la colaboración de la dirección del CS mediante contacto telefónico $\mathrm{y}$, posteriormente, se enviaron los cuestionarios para los/as MF seleccionados en su CS; b) para el 100\% de la muestra obtuvimos el tamaño del cupo y los pacientes/día en los Distritos Sanitarios.

Análisis estadístico. a) descriptivo de todas las variables, b) bivariante de la relación de todas las variables con el sexo; c) factorial exploratorio con extracción de componentes principales y rotación varimax, incluyendo los ítems con autovalores $>1$, evaluando la adecuación muestral con el índice de Kaiser-Meyer-Olkin (KMO) y el test de esfericidad de Barlett; d) fiabilidad de los factores con el alfa de Cronbach extrayendo el ítem; e) regresión múltiple para probar la validez convergente, para el conjunto de la muestra y por sexo. Usamos el método paso a paso, con probabilidad del $15 \%$ y de salida del 20\%. En los modelos se incluyeron las variables para las que existe diferencia estadísticamente significativa, por ello no se incluyen las de carga asistencial. Para controlar posibles efectos de relación entre las variables independientes se realizó un diagnóstico de colinealidad, que fue favorable sólo en el caso del primer factor para las variables "horas dedicadas a las tareas del hogar los sábados y domingos" y "quién realiza principalmente las tareas domésticas", por lo que ésta última fue excluida del modelo. 15.0 .

El paquete estadístico empleado fue SPSS

\section{RESULTADOS}

De las 500 personas estudiadas respondieron $368(73,6 \%), 182(71,7 \%)$ eran mujeres y $186(75,6 \%)$ hombres $(\mathrm{p}=0,316)$. Eran tutor/a 128 personas, de las que respondieron $101(78,9 \%)$ y no lo eran 372 de las que respondieron $267(71,8 \%)(p=0,114)$. Se 
Tabla 1

Cargas factoriales de las preguntas de la escala "relaciones familia-trabajo" $\mathrm{y}$ alfa de Cronbach de los factores $(\mathrm{N}=368)$

\begin{tabular}{|l|c|c|}
\hline \multicolumn{1}{|c|}{ Preguntas escala "relaciones familia-trabajo" } & $\begin{array}{c}\text { Sobrecarga y } \\
\text { déficit de } \\
\text { apoyo } \\
\text { familiar }\end{array}$ & $\begin{array}{c}\text { Conflicto } \\
\text { familia- } \\
\text { trabajo }\end{array}$ \\
\hline $\begin{array}{l}\text { Si falto algún día de casa las tareas domésticas siempre se } \\
\text { quedan sin hacer }\end{array}$ & 0,790 & \\
\hline Mi familia deja muchos asuntos de la casa para mí & 0,784 & \\
\hline $\begin{array}{l}\text { Me responsabilizo de la mayor parte de las tareas } \\
\text { domésticas }\end{array}$ & 0,779 & \\
\hline $\begin{array}{l}\text { Mi familia me agobia con cosas que deberían ser capaces } \\
\text { de hacer por si mismos }\end{array}$ & 0,675 & \\
\hline $\begin{array}{l}\text { Mi familia me quita parte del tiempo que me gustaría } \\
\text { dedicar al trabajo }\end{array}$ & 0,561 & \\
\hline A menudo necesitaría estar en el centro y en casa a la vez & 0,555 & \\
\hline $\begin{array}{l}\text { Mi horario de trabajo a menudo choca con mi vida } \\
\text { familiar }\end{array}$ & & 0,749 \\
\hline Estoy satisfecho/a con el tiempo que dedico a mi familia & & 0,728 \\
\hline Estoy satisfecho/a con el tiempo que dedico a mi trabajo & 0,669 \\
\hline $\begin{array}{l}\text { Mis obligaciones del trabajo y mis obligaciones en la } \\
\text { familia son compatibles }\end{array}$ & & 0,617 \\
\hline $\begin{array}{l}\text { Después del trabajo, llego a casa demasiado/a cansado/a } \\
\text { para hacer lo que me gustaría }\end{array}$ & & 0,543 \\
\hline Alfa de Cronbach & 0,817 & 0,724 \\
\hline
\end{tabular}

\section{Tabla 2}

Comparación por sexo de las variables independientes del estudio y de las dimensiones de la escala "relaciones familia-trabajo"

\begin{tabular}{|c|c|c|c|}
\hline Variables Cualitativas & $\begin{array}{l}\text { Médicas } \\
\mathrm{N}(\%)\end{array}$ & $\begin{array}{l}\text { Médicos } \\
\mathrm{N}(\%)\end{array}$ & $\mathrm{P}$ \\
\hline Especialidad MIRMF & $80(44,2 \%)$ & $62(33,33 \%)$ & 0,016 \\
\hline Vive solo/a con o sin hijos/as & $29(15,9 \%)$ & $9(4,8 \%)$ & 0,021 \\
\hline $\begin{array}{l}\text { Existen personas en el hogar con } \\
\text { cuidados especiales }\end{array}$ & $77(42,3 \%)$ & $79(42,5 \%)$ & 0,9738 \\
\hline La pareja trabaja fuera del hogar & $136(98,9 \%)$ & $123(78,8 \%)$ & $<0,001$ \\
\hline Tiene hijos/as & $152(83,5 \%)$ & $176(89,8 \%)$ & 0,077 \\
\hline $\begin{array}{lr}\begin{array}{l}\text { Las tareas domésticas las realiza } \\
\text { principalmente: }\end{array} & \\
& \text { La persona } \\
& \text { La pareja } \\
& \text { Otros }\end{array}$ & $\begin{array}{l}113(64,9 \%) \\
22(12,6 \%) \\
39(22,4 \%)\end{array}$ & $\begin{array}{l}58(33,5 \%) \\
97(56,1 \%) \\
18(10,4 \%)\end{array}$ & $<0,001$ \\
\hline Variables Cuantitativas & $\begin{array}{c}\text { Médicas } \\
\text { Media } \pm \text { SD }\end{array}$ & $\begin{array}{c}\text { Médicos } \\
\text { Media } \pm \text { SD }\end{array}$ & $\mathrm{P}$ \\
\hline Edad & $49,1 \pm 4,34$ & $51,3 \pm 4,86$ & $<0,001$ \\
\hline $\begin{array}{l}\text { Horas tareas domésticas lunes a } \\
\text { viernes }\end{array}$ & $2,9 \pm 3,09$ & $1,1 \pm 1,02$ & $<0,001$ \\
\hline $\begin{array}{l}\text { Horas tareas domésticas fines de } \\
\text { semana }\end{array}$ & $4,1 \pm 2,94$ & $1,9 \pm 1,62$ & $<0,001$ \\
\hline Cupo pacientes & $2055 \pm 224,80$ & $\begin{array}{l}2041 \pm \\
304,13\end{array}$ & 0,645 \\
\hline Pacientes/día consulta a demanda & $36,5 \pm 8,63$ & $36,4 \pm 9,28$ & 0,861 \\
\hline Dimensiones de la escala & $\begin{array}{c}\text { Médicas } \\
\text { Media } \pm \text { SD }\end{array}$ & $\begin{array}{c}\text { Médicos } \\
\text { Media } \pm \text { SD }\end{array}$ & $\mathrm{P}$ \\
\hline Sobrecarga y Déficit Apoyo Familiar & $19,82 \pm 8,19$ & $14,46 \pm 5,58$ & $8,109 \mathrm{e}-13$ \\
\hline Conflicto Familia-Trabajo & $17,96 \pm 5,60$ & $16,64 \pm 5,59$ & 0,024 \\
\hline
\end{tabular}




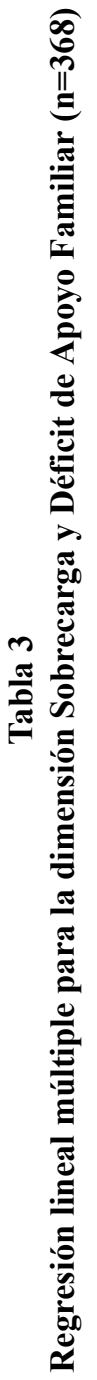

\begin{tabular}{|c|c|c|c|c|c|c|c|c|c|c|}
\hline & $\sim$ & $\begin{array}{l}8 \\
8 \\
8 \\
\overline{8}\end{array}$ & & & $\begin{array}{l}8 \\
8 \\
8 \\
8\end{array}$ & $\begin{array}{l}n \\
8 \\
0 \\
0\end{array}$ & $\begin{array}{l}\mathscr{8} \\
8 \\
0 \\
8\end{array}$ & & $\left|\begin{array}{l}0 \\
0 \\
0 \\
0\end{array}\right|$ & \\
\hline 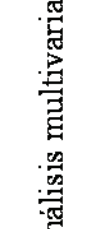 & 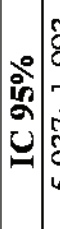 & م. & & & 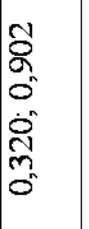 & $\begin{array}{l}8 \\
0 \\
0 \\
\infty \\
\infty \\
\tilde{N} \\
0 \\
0\end{array}$ & 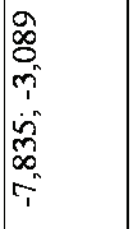 & & $\left|\begin{array}{c}0 \\
7 \\
7 \\
\dot{m} \\
m \\
0 \\
0 \\
0\end{array}\right|$ & \\
\hline & \begin{tabular}{lll}
$a$ \\
\hdashline
\end{tabular} & & & & $\vec{\sigma}$ & $\underset{\sim}{\stackrel{m}{\sim}}$ & 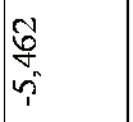 & & 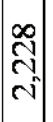 & \\
\hline & $=$ & & $\mid \begin{array}{c}\infty \\
8 \\
0 \\
0\end{array}$ & $\begin{array}{l}8 \\
8 \\
8 \\
8\end{array}$ & $\mid \begin{array}{l}\mathscr{8} \\
8 \\
8 \\
8 \\
\nabla\end{array}$ & $\begin{array}{l}m \\
\bar{\delta} \\
0 \\
0\end{array}$ & $\begin{array}{l}\mathscr{8} \\
8 \\
8 \\
8\end{array}$ & 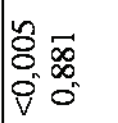 & $\begin{array}{l}m \\
= \\
0 \\
0\end{array}$ & 害 \\
\hline 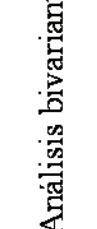 & 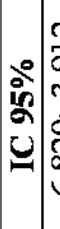 & 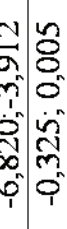 & 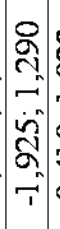 & 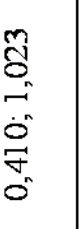 & 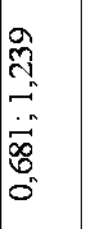 & $\begin{array}{l}\tilde{y} \\
0 \\
m \\
m \\
\tilde{m} \\
m \\
0 \\
0\end{array}$ & 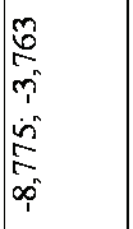 & 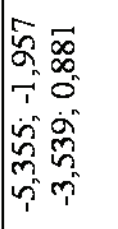 & $\left|\begin{array}{c}\infty \\
0 \\
0 \\
m \\
\dot{\hat{j}} \\
0 \\
0 \\
1\end{array}\right|$ & 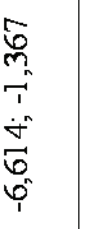 \\
\hline & 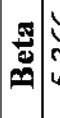 & 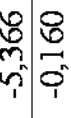 & $\begin{array}{c}n \\
m \\
0 \\
1\end{array}$ & & $\begin{array}{l}8 \\
8 \\
0 \\
0\end{array}$ & $\Rightarrow$ & $\begin{array}{l}8 \\
0 \\
01 \\
6 \\
1\end{array}$ & 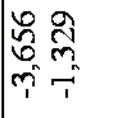 & $\begin{array}{l}\infty \\
\vdots \\
\vdots \\
-1\end{array}$ & $\begin{array}{l}\text { S. } \\
\text { mे }\end{array}$ \\
\hline 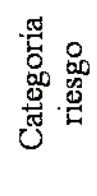 & & 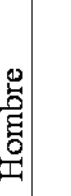 & $\mid \ddot{n}$ & & & $\ddot{n}$ & 总 & 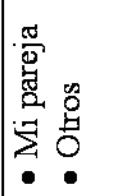 & in & 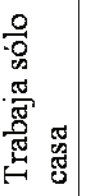 \\
\hline 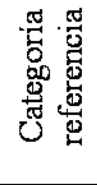 & & $\stackrel{5}{3}$ & 号 & & & $\stackrel{\circ}{2}$ & 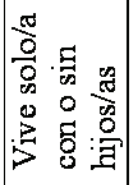 & $>^{\circ}$ & 㐒 & 苟 \\
\hline 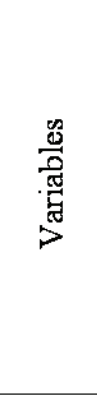 & & 丞 & 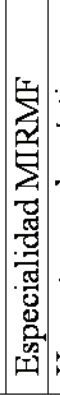 & 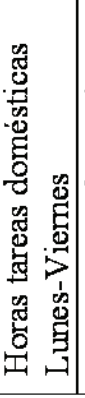 & 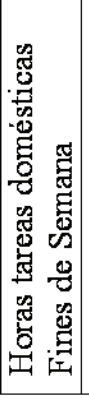 & 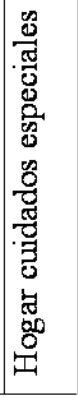 & 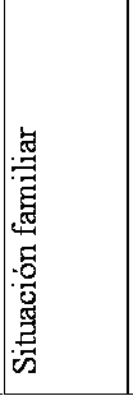 & 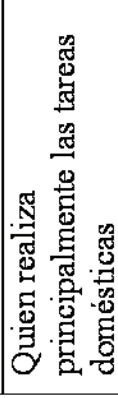 & 资 & 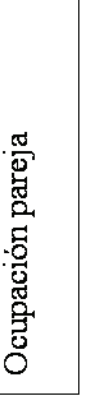 \\
\hline
\end{tabular}




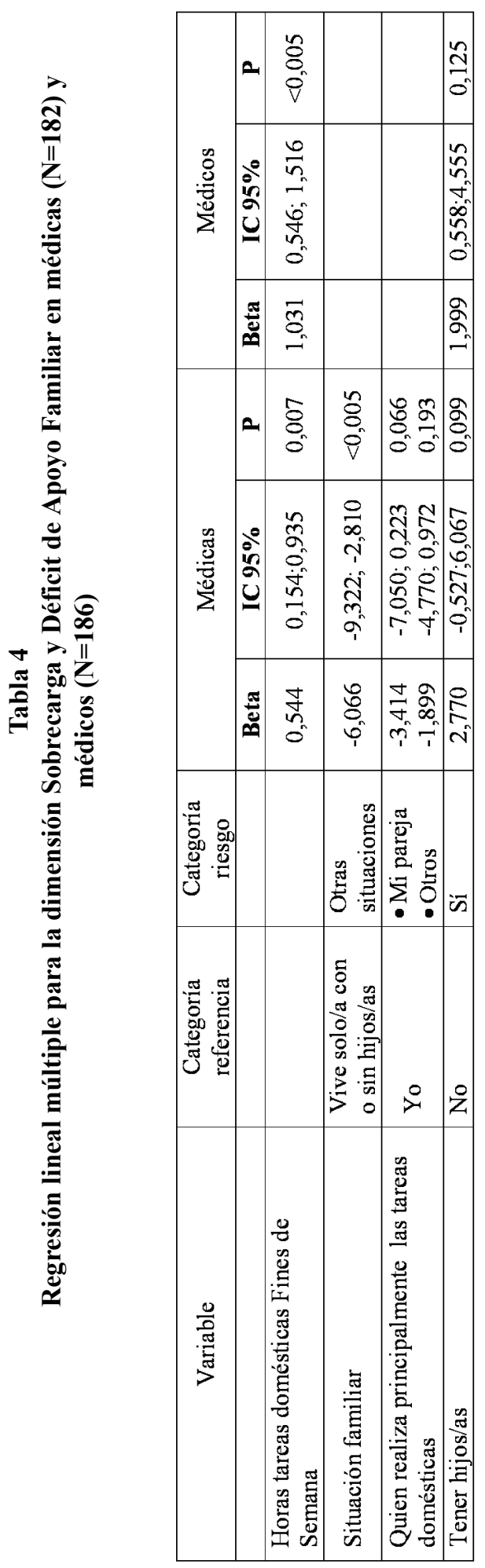




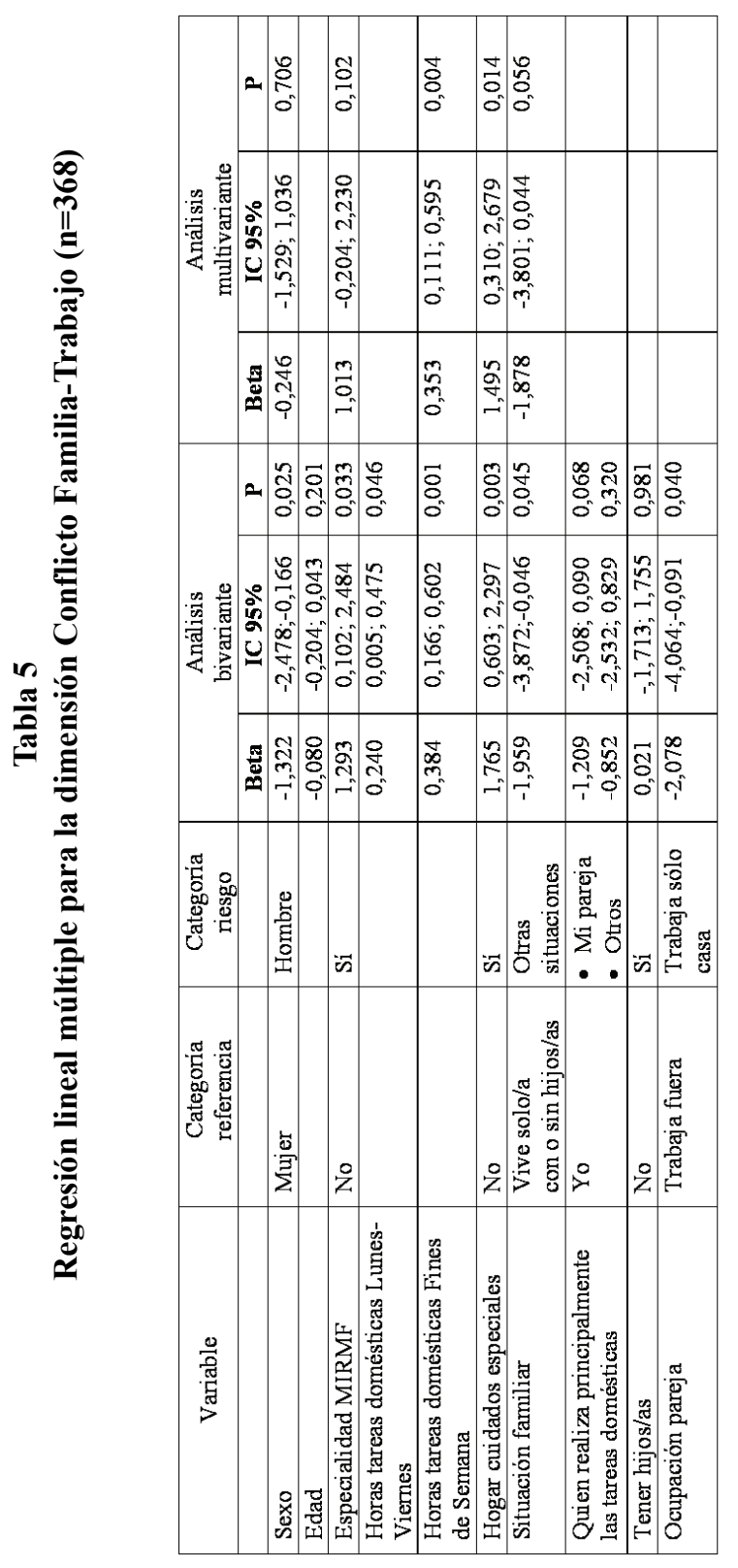




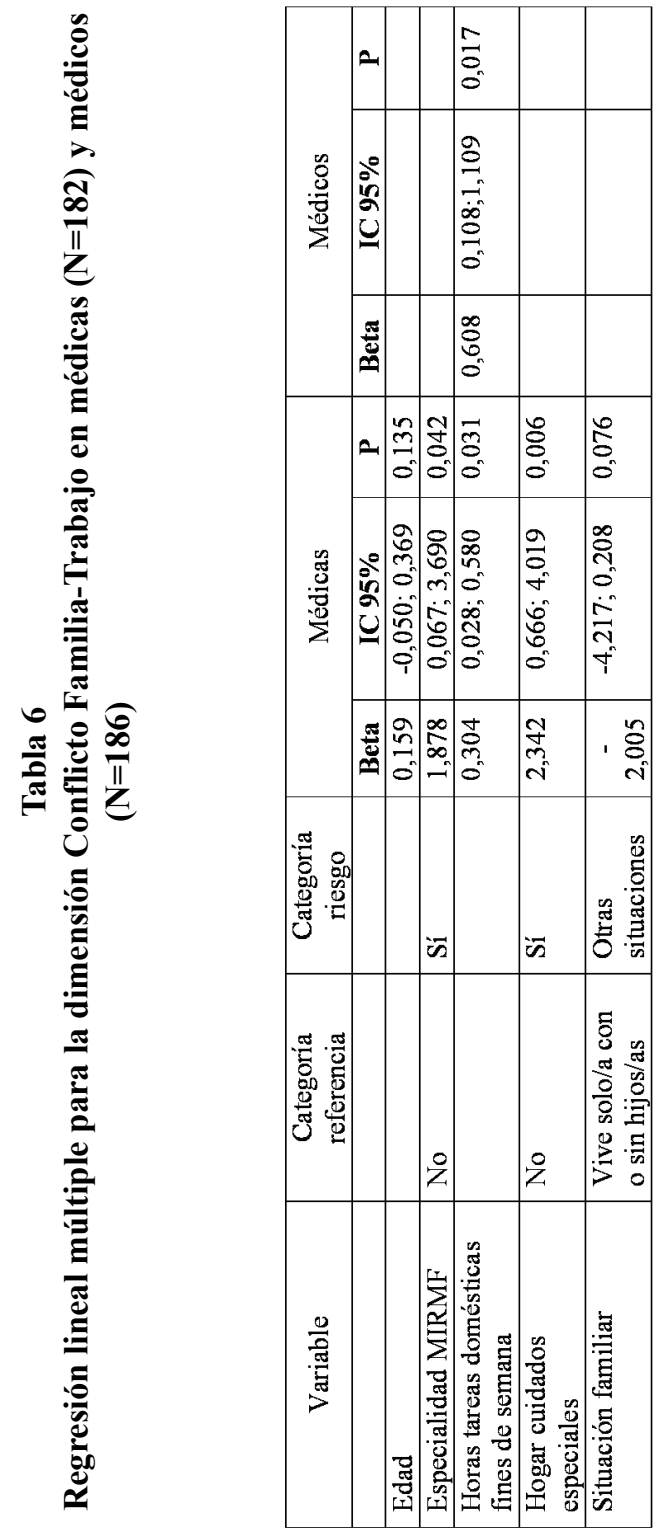


produjo un descenso de la potencia de la muestra al 60,3\%. La edad media global fue $50,18 \pm 4,74$ años.

El análisis factorial exploratorio identificó dos dimensiones que explicaron el 52,5\% de la varianza. La escala final tiene 11 preguntas (anexo 1). Las cargas factoriales y el alfa de Cronbach de ambos factores aparecen en la tabla 1.

El primer factor lo llamamos Sobrecarga y Déficit de Apoyo Familiar (SDAF) e integra 6 preguntas sobre falta de ayuda y colaboración de la familia para realizar las tareas domésticas. El alfa de Cronbach de este factor fue 0,817 .

Al segundo factor lo llamamos Conflicto Familia-Trabajo (CFT) y contiene 5 preguntas que reflejaron el compromiso entre la dedicación a la familia y al trabajo junto al grado de dificultad para hacer compatibles ambas esferas. El alfa de Cronbach del factor fue 0,724 .

Comparando las variables estudiadas por sexo (tabla 2), destaca que las mujeres son más jóvenes y tienen con más frecuencia formación MIRMF, dedican más horas a tareas del hogar, viven solas, con o sin hijos, en mayor medida que los médicos, y casi el doble de médicas que de médicos realizan solas las tareas domésticas. No existen diferencias estadísticamente significativas en las variables de carga asistencial. Se observó que las médicas percibían mayor Sobrecarga y Déficit Apoyo Familiar y expresaban mayor Conflicto Familia- Trabajo.

La tabla 3 muestra las relaciones crudas y ajustadas de todas las variables con la SDAF para el conjunto de la muestra. Después del ajuste el SDAF era superior en las médicas, en quienes dedican más horas a tareas domésticas el fin de semana, conviven con personas que requieren cuidados especiales, viven solo/a, frente al resto de situaciones familiares, y tienen descendientes en el hogar.
Al analizar por sexo (tabla 4) se observó que hay cuatro variables familiares que se relacionan con el SDAF en las médicas: horas dedicadas a tareas domésticas el fin de semana, vivir sola, realizar las tareas domésticas, y tener hijos/as. Sin embargo en los médicos sólo aumenta el SDAF al hacerlo las horas dedicadas a tareas domésticas el fin de semana.

En el conjunto de la muestra el CFT (tabla 5 ) es superior en las médicas, en quienes dedican más horas a tareas domésticas el fin de semana, y conviven con personas que requieren cuidados especiales. Por sexo (tabla 6) se ve que las médicas perciben más CFT al dedicar más horas a tareas domésticas el fin de semana, vivir sola, convivir con personas que requieren cuidados especiales, y ser MIRMF. Para los médicos sólo las horas dedicadas a tareas domésticas el fin de semana guardan relación positiva con el CFT.

\section{DISCUSIÓN}

La tasa de respuesta fue buena tratándose de un cuestionario autoadministrado. Entre los que respondieron y los que no respondieron no hubo diferencias estadísticamente significativas por sexo ni por ser tutor/a, lo que permite pensar en la ausencia de sesgos de selección atribuibles a la respuesta.

El conjunto de MF que trabajan en medio urbano percibe la existencia de dos dimensiones diferenciadas en las relaciones familia-trabajo, la primera dimensión empírica está relacionada con la sobrecarga de tareas del hogar y la falta de apoyo de la familia para el desarrollo de las mismas, y la segunda está referida a la vivencia de conflicto para compatibilizar los roles de las esferas familiar y laboral. En ambos factores las médicas alcanzan puntuaciones superiores a sus compañeros.

La correlación observada entre las variables de carga familiar y el SDAF es una 
medida de la validez convergente de la escala, las médicas puntúan más alto en SDAF y asumen mayores cargas familiares y responsabilidades del hogar sin recibir ayuda. Los resultados muestran que las médicas asumen más responsabilidades familiares que los médicos, algo similar a lo que ocurre en la población general, en la que en 2003 las mujeres dedicaban a las tareas domésticas 5,47 horas/día y los hombres 2,22, desigualdad que persistía al analizar por edad, situación familiar, y nivel educativo ${ }^{21}$. Además, más médicas que médicos realizan solas las tareas domésticas, lo que también concuerda con datos de población general de $2008^{10}$. Es superior el número de médicas que viven solas con o sin hijos, diferencia que también se observaba en población andaluza en 2005, en la que había $29 \%$ mujeres viviendo solas con o sin descendencia frente a $15,6 \%$ de hombres $^{22}$. Más médicas tienen pareja trabajando fuera del hogar, y la ocupación de las parejas de médicos y médicas es diferente ${ }^{23}$. Datos similares en estas variables familiares se observan en otros lugares y en diversas especialidades médicas ${ }^{24,25}$. Es importante señalar que en nuestro estudio la diferencia por sexo aparece entre vivir solo/a versus vivir en pareja, y ello es lo que se relaciona con las dos dimensiones de la escala, sin embargo no existen diferencias por sexo entre tener o no tener hijos/as, independientemente de que se conviva o no con pareja, por tanto, lo relevante para percibir mayor SDAF y más CFT es no convivir con otra persona adulta.

Estas dos dimensiones expresan la percepción subjetiva del conflicto que siente una persona para conciliar dos esferas de su vida y parece que vivir con otra persona adulta conlleva la sensación de compartir con ella y/o recibir apoyo para conciliar las propias responsabilidades domésticas. Se ha destacado el papel de la pareja como fuente de apoyo para que las mujeres profesionales desempeñen su carrera ${ }^{26}$. Concretamente las médicas en pareja tienen mayores probabilidades de tener una especialización médica ${ }^{25}$ y perciben mayor apoyo para su desarrollo profesional $^{27}$.

Las médicas perciben mayor SDAF, lo que se relaciona con cuatro características familiares: las horas dedicadas a las tareas del hogar los fines de semana, vivir solas, realizar las tareas domésticas sola, y convivir con hijos/as. Sin embargo, en los médicos la percepción de SDAF aumenta solo al hacerlo las horas dedicadas al hogar los fines de semana. Esto significa que el efecto del trabajo en la familia es más importante y más complejo en el caso de las mujeres, posiblemente porque eluden con mayor dificultad que los hombres el desempeño de esas funciones. Ello confirma que las relaciones familia-trabajo están moduladas por la construcción social de género ${ }^{12}$.

Las médicas de la muestra perciben mayor CFT que los médicos, lo que también ocurre en otros ámbitos ${ }^{28}$, y ello puede implicar cambios en su carrera ${ }^{25,29}$. Entre médicos y médicas alemanes la interferencia del trabajo en la familia no produce diferencias por sexo en el conflicto percibido ${ }^{30}$.

En nuestro estudio, el CFT se relaciona con características diferentes según sexo; en los médicos solo las horas dedicadas al hogar los fines de semana incrementan la percepción de conflicto, en las médicas la percepción de conflicto es más compleja y se asocia con tres variables de carga familiar y con ser MIRMF. Parece que esta formación supone una cierta actitud adquirida y/o reforzada que, por alguna razón, aumenta el conflicto, quizás al aumentar la autoexigencia profesional. En población aragonesa el nivel de estudios también se relaciona con el conflicto $^{31}$.

La diferente complejidad de factores relacionados con la SDAF y con el CFT según el sexo pone de manifiesto que sus causas, consecuencias y percepciones son diferentes para mujeres y hombres ${ }^{12,14,15}$. Según una revisión de 190 estudios con población tra- 
bajadora, los hallazgos sobre la existencia de conflicto son inconsistentes, pero los mecanismos son diferentes entre mujeres y hombres $^{32}$.

Además de subyacer mecanismos diferentes en el funcionamiento de la relaciones familia-trabajo, las consecuencias de las mismas son mayores en la carrera de las médicas que en la de los médicos ${ }^{19,25,29,33}$.

Para comprender en profundidad estas diferencias de sexo y las consecuentes desigualdades de género en el trabajo es necesario contar con la teoría de género. El género es un sistema de relaciones asimétricas constituido por tres niveles: estructural -división sexual del trabajo o lugar que ocupan mujeres y hombres-, simbólico -ideas y discursos sociales sobre los sexos-, y subjetivo -identidad de hombres y mujeres- ${ }^{34}$. Estos tres planos interrelacionados actúan manteniendo la complejidad de las relaciones entre familia y trabajo en cada sexo.

Las desigualdades de género en el trabajo dejaran de ser un problema cuando las médicas no se vean obligadas a elegir entre las responsabilidades familiares y el estatus profesional ${ }^{35}$, lo que se producirá sólo cuando los hombres se incorporen en iguales condiciones a las responsabilidades familiares. Se ha señalado que existe un cambio generacional incipiente en hombres y mujeres, quienes desean poner límites al tiempo de trabajo y disponer de más tiempo para la familia, lo que representa una oportunidad para desafiar las normas masculinas del mercado de trabajo ${ }^{36}$.

La investigación presenta fortalezas y limitaciones; entre las primeras está que el estudio de variables asistenciales, algunas características personales, familiares y profesionales, nos permite controlar el efecto de todas ellas en la explicación de la SDAF y del CFT. Por otra parte, el análisis por sexo arroja luz sobre las diferencias en los mecanismos que subyacen en médicas y médicos por separado.
Las limitaciones del estudio son, en primer lugar, que la muestra es de CS y MF del medio urbano, por lo que los resultados no son extrapolables al medio rural, aunque es probable que quienes trabajan en ese entorno tengan más de común que de diferente con quienes lo hacen en CS urbanos en su percepción de las relaciones familia-trabajo, entre otras razones, porque su ubicación profesional actual en medio urbano es independiente de que hayan nacido en medio rural o no y, por tanto, también de los aspectos más característicos de la socialización de género de ambos entornos. No obstante la escala obtenida podría ser de nuevo validada en ese subgrupo. El diseño trasversal de la investigación impide conocer la dirección de las relaciones estudiadas, lo que requiere estudios longitudinales para su confirmación.

Existen instrumentos más complejos y extensos para medir las relaciones familiatrabajo, pero nuestro estudio pretendía diseñar una escala breve, adaptada al entorno de la profesión médica, y útil para los objetivos más amplios de la investigación. La escala obtenida es válida, fiable y recoge dos dimensiones de las relaciones entre vida familiar y laboral. Podría ser de interés desarrollar instrumentos que evaluaran no sólo la vertiente negativa o de conflicto sino también los aspectos positivos o facilitadores, así como la dirección de las relaciones existentes entre ambas esferas de la vida.

\section{AGRADECIMIENTOS}

A la Consejería de Salud de la Junta de Andalucía, al Fondo de Investigación Sanitaria y a los Fondos FEDER de la Unión Europea, por la ayuda concedida para la investigación. A las médicas y médicos de familia por su colaboración.

\section{BIBLIOGRAFÍA}

1. Ortiz Gómez T. La práctica sanitaria en la historia ¿una cuestión femenina? Eidon. Rev Fundación de Ciencias salud. 2007, 25: 61-65. 
2. Instituto Nacional de Estadística. Profesionales sanitarios colegiados año 2009. Disponible en: http://www.ine.es/jaxi/menu.do?type=pcaxis\&path $=/ \mathrm{t}$ $15 / \mathrm{p} 416 \&$ file $=$ inebase $\& \mathrm{~L}=0$

3. Elston MA. Women and medicine: The future. Londres: Royal College of physician; 2009.

4. Observatorio de salud de la mujer. Informe salud y género 2007-2008. Mujeres y hombres en las profesiones sanitarias. Madrid: Ministerio de Sanidad y Consumo; 2009 .

5.Rohlfs I, Arrizabalaga P, Artazcoz L, Borrel C, Fuentes M, Valls-Llobet C. Salud, estilos de vida y condiciones de trabajo de los médicos y médicas de Cataluña. Barcelona: Fundación Galatea; 2007

6.Santamaría A, Merino A, Viñas O, Arrizabalaga P. Does Medicine still show an unresolved discrimination against women? Experience in Two European University Hospitals. J Med Ethics. 2009, 35: 104-106.

7. Jagsi R, Guancial E, Cooper Worobey C, Henault L, Chang Y, Starr R et al. The "gender gap" in authorship of academic medical literature. A 35-year perspective. N. Engl J Med. 2006, 355: 281-287.

8. Ortiz Gómez T, Birriel Salcedo J, Ortega del Olmo R. Género, profesiones sanitarias y salud pública. Gac Sanit. 2004, 18: 189-194.

9.Schiaffino A, Garcia M, Fernandez E. Autoría y presentación de resultados según el género en cuatro revistas biomédicas españolas. Gac Sanit. 2001, 15: 251254.

10. Centro de Investigaciones Sociológicas. Estudio 2766. Barómetro de junio de 2008; 2008. Disponible en: http://217.140.16.67/cis/opencms/-Archivos/Marginales/2760_2779/2766/Cru2766_enlace.html.

11.Servicio Andaluz de Salud. Mujeres y hombres en los indicadores sanitarios. SAS Información. 2008, 38: 5-8.

12. Martínez-Pérez MD, Osca A. Estudio psicométrico de la versión española de la escala de "Conflicto Familia-Trabajo" de Kopelman, Greenhaus y Connoly (1983). Rev Psicol Soc. 2001, 16: 43-57.

13. Xu L. View on work-family linkage and workfamily conflict model. Int $\mathrm{J}$ of Business and Management. 2009, 4: 229-233.

14. Greenhaus JH, Beutell N. Sources of conflict between work and family roles. Acad Manage Rev. 1985, 10: $76-88$
15. Van Steenbergen E, Ellemers N, Mooijaart A. How work and family can facilitate each other: distinct types of work-family facilitation and outcomes for women and men. J Occup Health Psychol. 2007, 12: 279-300.

16. Barnett R, Hyde J.S. Women, men, work, and family. Am Psychol. 2001, 56: 781-796.

17 .Martínez-Pérez MD, Osca Segovia A. Análisis psicométrico del Inventario de Apoyo Familiar para Trabajadores. Psicothema. 2002, 14: 310-316.

18. Moncada i Lluis S, Llorens C, Kristensen S. Método Istas 21 (CoPsoQ) Manual para la evaluación de riesgos psicosociales en el trabajo. Madrid: Instituto sindical de Trabajo, Ambiente y Salud; 2004.

19.Verlander G. Female physicians: balancing career and family. Acad Psychiatry. 2004, 28: 331-336.

20. Allen T, Herst D, Bruck C, Sutton M. Consequences associated with work-to-family conflict: A review and agenda for future research. J Occup Health Psychol. 2000, 5: 279-308.

21. Instituto Nacional de Estadística. Encuesta de empleo de tiempo 2002-2003. Disponible en: http://www.ine.es/jaxi/menu.do?type $=$ pcaxis\&path $=\%$ $2 \mathrm{Ft} 25 \% 2 \mathrm{Fe} 447 \&$ file $=$ inebase $\& \mathrm{~L}=0$

22.Instituto de Estadística de Andalucía. Anuario estadístico de Andalucía 2006. Perspectivas de género. Sevilla: Instituto de Estadística de Andalucía; 2006.

23.Delgado A, Saletti-Cuesta L, López-Fernandez LA, Luna JD, Mateo I, Jiménez JM. Características de la familia de origen y de la familia formada por las médicas y los médicos de familia de Andalucía. Rev Esp Salud Pública. 2009, 83: 891- 897.

24.Danesh-Meyer H, Deva N, Ku JYF, Carroll SC, Tan YW. Gamble G. Differences in practice and personal profiles between male and female ophthalmologists. Clin Experiment Ophthalmol. 2007, 35: 318-323.

25. Gjerberg E. Women doctors in Norway: the challenging balance between career and family life. Soc Sci Med. 2003, 57: 1327-1341.

26.Dio Bleichmar E. ¿Todas Madame Curie? Subjetividad e identidad de la científicas y tecnólogas. Rev Aperturas Psicoanalíticas. 2006, 24. Disponible en: http://www.aperturas.org/articulos.php?id=0000419\&a $=$ Todas-Madame-Curie-Subjetividad-e-identidad-delas-cientificas-y-tecnologas

27. Schrager S, Kolan A, Dottl S. Is that your pager or mine: A survey of women academic family physicians 
in dual physician families. Wis Med J. 2007; 106 (5): 251-255.

28. Mobilos S, Chan M, Belle Brown M. Women in medicine. The challenge of finding balance. Can Fam Physician. 2008, 54: 1285-6e1-5.

29. Warde C, Allen W, Gelberg L. Physician role conflict and resulting career changes. Gender and generational differences. J Gen Intern Med. 1996, 11: 729-35.

30. Fub I, Nubling M, Hasselhorn HM, Schwappach M, Rieger M. Working conditions and work-family conflict in German hospital physicians: psychological and organisational predictors and consequences. BMC Public Health. 2008, 8: 353 doi:10.1186/1471-2458-8353.

31. De Luis Carnicer MP, Martínez Sánchez A, Pérez Pérez M, Vela Jiménez MJ. Work- family conflict in a southern European country. J Manag Psychol. 2004, 19: 466-489.

32.Eby LT, Casper WJ, Lockwood A, Bordeaux C, Brinley A. Work and family research in IO/OB: Content analysis and review of the literature (1980-2002). J Vocat Behav. 2005, 66: 124-197.

33. Shollen L, Bland C, Finstad D, Taylor A. Organizational climate and family life: How these factors affect the status of women faculty at one medical school. Acad Med. 2009, 84: 87-91.

34. Harding S. Ciencia y feminismo. Madrid: Morata; 1996.

35.Heath I. Women in medicine. BMJ. 2004, 329: 412-3

36.Williams J. Unbending gender. Why family and work conflict and what to do about it. New York: Oxford University Press; 2000. 
Anexo 1

Escala relaciones familia-trabajo

\begin{tabular}{|l|l|l|l|l|l|l|}
\hline \multicolumn{1}{|c|}{ Preguntas } & $\begin{array}{c}\text { Total } \\
\text { desacuerdo }\end{array}$ & 2 & 3 & 4 & 5 & $\begin{array}{c}6 \\
\text { Total } \\
\text { acuerdo }\end{array}$ \\
\hline $\begin{array}{l}\text { Si falto algún día de casa las tareas domésticas siempre se } \\
\text { quedan sin hacer }\end{array}$ & & & & & & \\
\hline Mi familia deja muchos asuntos de la casa para mí & & & & & & \\
\hline $\begin{array}{l}\text { Me responsabilizo de la mayor parte de las tareas } \\
\text { domésticas }\end{array}$ & & & & & & \\
\hline $\begin{array}{l}\text { Mi familia me agobia con cosas que deberían ser capaces } \\
\text { de hacer por si mismos }\end{array}$ & & & & & & \\
\hline $\begin{array}{l}\text { Mi familia me quita parte del tiempo que me gustaría } \\
\text { dedicar al trabajo }\end{array}$ & & & & & & \\
\hline A menudo necesitaría estar en el centro y en casa a la vez & & & & & \\
\hline $\begin{array}{l}\text { Mi horario de trabajo a menudo choca con mi vida } \\
\text { familiar }\end{array}$ & & & & & & \\
\hline Estoy satisfecho/a con el tiempo que dedico a familia & & & & & & \\
\hline Estoy satisfecho/a con el tiempo que dedico a mi trabajo & & & & & & \\
\hline $\begin{array}{l}\text { Mis obligaciones del trabajo y mis obligaciones en la } \\
\text { familia son compatibles }\end{array}$ & & & & & \\
\hline $\begin{array}{l}\text { Después del trabajo, llego a casa demasiado/a cansado/a } \\
\text { para hacer lo que me gustaría }\end{array}$ & & & & & \\
\hline
\end{tabular}

